\title{
Erratum to: Best proximity point results for MK-proximal contractions on ordered sets
}

\author{
Mohamed Jleli and Bessem Samet
}

J. Fixed Point Theory Appl.

DOI 10.1007/s11784-013-0125-4

The correct Acknowledgement for this article should read as follows.

\section{Acknowledgement}

This project was supported by King Saud University, Deanship of Scientific Research, College of Science Research Center

Mohamed Jleli

Department of Mathematics

King Saud University, Riyadh

Saudi Arabia

e-mail: jleli@ksu.edu.sa

Bessem Samet

Department of Mathematics

King Saud University, Riyadh

Saudi Arabia

e-mail: bsamet@ksu.edu.sa 